\title{
ENHANCEMENT OF EPILEPTIFORM ACTIVITY IN THE EEG BY 3-D ADAPTIVE SPATIAL FILTERING: SIMULATIONS AND REAL DATA
}

\author{
Donna-Maree Ward ${ }^{1}$, Student Member, IEEE, Richard D. Jones ${ }^{2,3^{*}}$, Senior Member, IEEE, \\ Philip J Bones ${ }^{1}$, Senior Member, IEEE, Grant J. Carroll ${ }^{4}$ \\ ${ }^{1}$ Department of Electrical and Electronic Engineering, University of Canterbury \\ 2 Department of Medical Physics \& Bioengineering, Christchurch Hospital \\ ${ }^{3}$ Department of Medicine, Christchurch School of Medicine \\ ${ }^{4}$ Department of Neurology, Christchurch Hospital \\ Christchurch, New Zealand
}

\begin{abstract}
An adaptive 'beamformer' spatial filter has been investigated for the enhancement of signals from deep sources in the brain suspected of being epileptiform discharges (EDs). A forward 3-D inhomogeneous spherical model that relates a dipolar source to recorded signals is used to determine the beamformer's spatial response constraints. The beamformer adapts, using the least mean squares algorithm, to reduce signals from sources distant from the assumed source location and produces three outputs, the orthogonal components of the signal estimated to have arisen at or near the assumed location. Simulations were performed by using the same forward model to superimpose realistic EDs on normal EEG recordings. The simulations showed that the beamformer could enhance signals emanating from epileptogenic foci by a mean $120 \%$. The beamformer was also shown to be relatively insensitive to depth and to inaccuracies in estimate of location (depth and angular location) of both radially and tangentially oriented dipoles. A preliminary clinical evaluation on real EEG records showed that the beamformer was able to enhance definite EDs by a mean $102 \%$. Overall, these results demonstrate the value of the beamformer as a non-invasive means of enhancing activity from epileptogenic foci whose location is ill-defined.
\end{abstract}

\section{INTRODUCTION}

$\mathrm{T}_{\mathrm{i}}$ $\mathrm{HE}$ detection of epileptiform discharges (EDs) in the EEG is an important component in the diagnosis of epilepsy. However, when the epileptogenic source is located deep in the brain, the EDs at the scalp are often masked by more superficial, higher amplitude EEG activity. Currently, these can only be investigated through invasive electrophysiological investigations such as sphenoidal electrodes, depth electrodes, and electrocorticography, or extremely expensive procedures such as MEG.

Lopes da Silva et al. [1] used autoregressive filtering to effectively enhance non-stationarities, including EDs, in the EEG. Similarly, James et al. [2] used multireference adaptive noise cancelling to enhance non-stationarities by means of a multilayer perceptron ANN.

*E-mail: r.jones@chmeds.ac.nz
Provided there exists some a priori clinical evidence indicating the approximate location of an epileptogenic source, this information can be used to enhance any EDs coming from this region of the brain and attenuate EEG from other areas of the brain. This is known as 3-D spatial filtering, or 'beamforming', because of its origin in applications such as radar, sonar, geophysical and astrophysical exploration. We employ an adaptive beamformer, described by Van Veen et al. [3], Spencer et al. [4], van Drongelen at al. [5], and Ward [6], to enhance signals from deep sources in the brain suspected of containing epileptogenic sources, while simultaneously attenuating signals from other focal or distributed sources within the brain and some types of artifact.

This paper presents (a) aspects of a comprehensive investigation of the performance characteristics of the beamforming technique through the use of simulated dipolar EDs at specified sites in the brain, and (b) some preliminary results demonstrating the enhancement of EDs in real EEG data.

\section{The Beamforming Technique}

The beamformer used to process EEG signals is depicted in Fig. 1. This particular design is known as either an adaptive linearly constrained minimum variance (LCMV) beamformer or a generalized sidelobe canceller (GSC) [4], [7]. It has the effect of preserving the signal arising from the chosen source location while minimizing the contributions from other sources. In the EEG case, we have 16 channels of input $\mathbf{v}(k)$ to the beamformer and a single output channel $y(k)$. The beamformer is composed of two segments: (1) a non-adaptive section which contains a set of fixed weights $\mathbf{w}_{\mathrm{f}}$ which implement a spatial filter on the input EEG data, and (2) an adaptive section comprising a fixed blocking matrix $\mathbf{B}_{\mathrm{n}}$ and a set of variable weights $\mathbf{w}_{\mathrm{n}}(k)$. The role of the blocking matrix is to block signals arising from the location of interest while allowing signals from other locations to pass through as the reduced dimensional vector $\mathbf{u}(k)$. The least mean squares (LMS) algorithm is used to update the set of weights $\mathbf{w}_{\mathrm{n}}(k)$. The scalar result of multiplying $\mathbf{u}(k)$ by the adapted weights is 


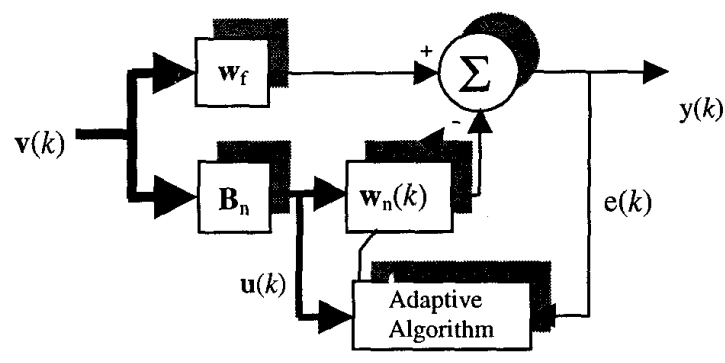

Fig. 1. Adaptive LCMV Beamformer.

then subtracted from the non-adaptive section output to cancel signals from outside the location of interest. Consequently, the output $y(k)$ is a single channel representing an estimate of the signal emanating from an equivalent dipolar source at a chosen location and orientation. Three such beamformers can be combined to resolve the source orientation, one for each of the three orthogonal directions $x, y$, and $z$.

\section{Simulations}

Data for the simulation studies was obtained from routine EEG recordings at Christchurch Hospital. Sixteen channels of EEG were recorded on the ipsilateral-ears referential montage from electrodes placed according to the 10-20 International System. Each channel of the recorded EEG was low-pass filtered at $70 \mathrm{~Hz}(100 \mathrm{~dB} / \mathrm{dec})$, sampled at $200 \mathrm{~Hz}$, and digitized to 12-bit precision.

EDs and normal background EEG were extracted from epileptiform and normal EEG recordings respectively to facilitate controlled investigation of the beamformer's performance and characteristics on simulated data. Each simulated epoch of data was arbitrarily chosen to be of $4 \mathrm{~s}$ ( 800 samples) duration since the beamformer required of the order of $2 \mathrm{~s}$ to adapt.

EEG recordings from patients with focal epilepsy were viewed by an EEGer for definite EDs. These EDs were then extracted from a single channel of the recorded EEG. The EDs were selected to represent the amplitude and morphology of EDs to be used as deep dipolar foci in simulation studies. The EDs selected included both monophasic and biphasic EDs of variable duration but were typically less than $140 \mathrm{~ms}$. The peak of the extracted ED was sited at 700 samples and padded with zeros to 800 samples. For all simulation studies, one monophasic and one biphasic ED, extracted from different patients, were each superimposed upon five background EEG records from different patients to yield ten sets of EEG data.

The isolated EDs were used as the amplitudes of simulated current dipoles at arbitrary fixed locations and orientations in the head. The location is specified by polar angle $\theta$, azimuthal angle $\phi$, and eccentricity $e$ (i.e., normalized depth of the source). A forward 3-layered inhomogeneous and isotropic spherical model [8] was applied to determine the potential distribution on the surface of the scalp at the 16 electrode positions. The model consisted of three concentric spheres representing the scalp, skull, and brain (Fig. 2). The radii of these were $92 \mathrm{~mm}, 85 \mathrm{~mm}$, and $80 \mathrm{~mm}$ respectively and the relative conductivity of the scalp and brain to that of the skull was 80 [9].

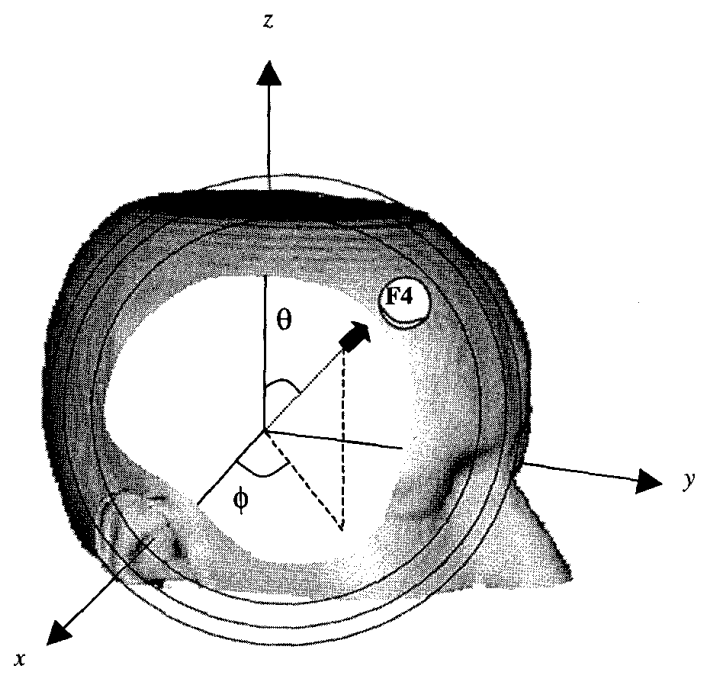

Fig. 2. Location and direction of deep epileptic source.

The 16 channels of voltage determined at each electrode (with respect to reference electrodes $\mathrm{A} 1$ and $\mathrm{A} 2$ ) were then superimposed upon one of several normal background EEG recordings to yield multichannel scalp EEG $\mathbf{v}(k)(16 \times 800)$ containing simulated spikes from dipoles located anywhere in the brain. This represented the EEG record to be input to the beamformer.

Different recording montages can be handled by the beamformer by premultiplying the input to the beamformer by an appropriate montage matrix.

In addition to visualizing the beamformer's output $y(k)$ in any coordinate or resultant direction, we use the forward model to project the beamformer output to the surface of the scalp at standard 10-20 electrode sites and compute corresponding referential or bipolar channel signals as required.

\section{Measures of Performance}

In order to quantify improvements in SNR due to the beamformer, we define ar enhancement ratio ER as

$$
\mathrm{ER}=\mathrm{SNR}_{o j} / \mathrm{SNR}_{i j}
$$

where $\mathrm{SNR}_{o j}$ is the adapted output inline signal, $\mathrm{SNR}_{i j}$ is the input signal, and $j$ corresponds to the input channel containing the most prominent $\mathrm{ED}$ (i.e., the highest $\mathrm{SNR}_{i j}$ ), where $j=1 . .16$. The adapted output inline signal arises when the beamformer is placed with the same orientation as that of the 
dipolar source, referred to as the 'inline beamformer'. Both $\mathrm{SNR}_{o j}$ and $\mathrm{SNR}_{i j}$ are defined as the ratio of the RMS amplitude of 5 samples $(25 \mathrm{~ms}$ ), centred on the peak of the spike, to the RMS amplitude of the background, also centred on the peak of the spike, over a $1 \mathrm{~s}$ interval. In all simulations the ER was determined by the inline beamformer.

In the case of the projected output, an ER based on a single-channel comparison is inadequate since the epileptiform event is enhanced to varying degrees on several channels. Therefore, a global ER performance measure is required. We have chosen to use the mean enhancement ratio $\overline{E R}$ calculated from the $\mathrm{ER}$ for each of the 16 channels. However, because estimates of SNR become inaccurate for low signal levels, input channels with very low SNRs can lead to erroneous ERs and, hence, an inaccurate $\overline{\mathrm{ER}}$. To overcome this, we have chosen to use an arbitrary (based on observations made with an EEGer) SNR threshold of 0.7 . Thus, any channel (input or output) which has a $S N R<0.7$ is excluded from the calculation of $\overline{\mathrm{ER}}$.

\section{Simulations RESUlts}

\section{A. Enhancement of EDs}

Overall, the simulations demonstrated the beamformer's ability to enhance signals emanating from epileptogenic foci by a mean $120 \%(-30 \%-360 \%)$.

\section{B. Dependence of ER on Signal Magnitude}

The performance of the beamformer was investigated to determine whether the ER was dependent on the absolute magnitude of the epileptiform signal. Preliminary results for both monophasic and biphasic sources superimposed upon the same segment of noisy background EEG, showed that the ER was independent of the size of the ED.

\section{Sensitivity to Depth of Beamformer}

The effect of inaccuracy in supposed depth of the dipole source on beamformer performance was investigated. In this, the actual dipole (solid arrow) was at the location indicated while the location of the estimated dipole (unfilled arrow) was

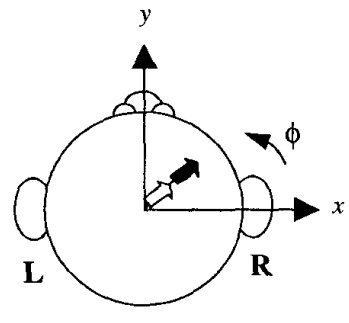

Top view

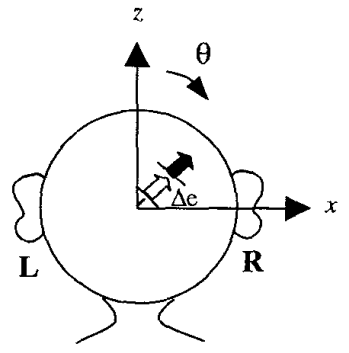

Back view
Fig. 3. Location of actual dipole (solid arrow) and estimated dipole (unfilled arrow). Orientation was constant in both cases. obtained by translation along a radial direction, a distance $\Delta e$ from the actual dipole (Fig. 3).

Beamforming at the location of the estimated dipole produced the results displayed in Fig. 4, in which the beamformer's performance was shown to be reasonably insensitive to the depth of the estimated dipole.

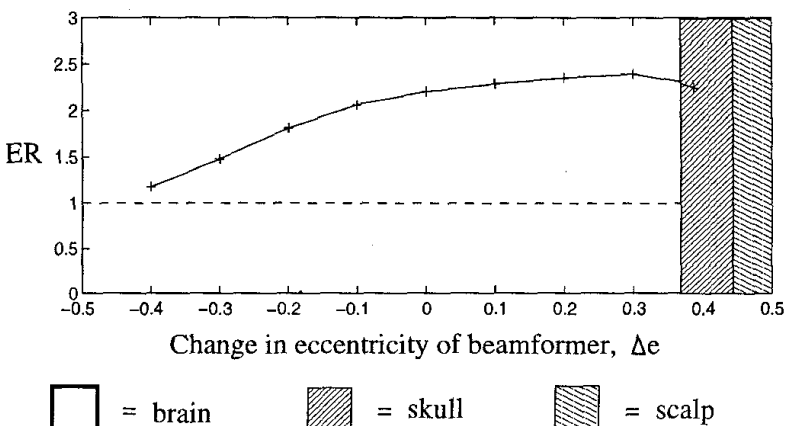

Fig. 4. Sensitivity to inaccuracies in depth of beamformer with repeat to the actual dipole.

\section{Sensitivity to Polar Location of Beamformer}

Sensitivity of the beamformer to inaccuracy in location of dipole source with respect to polar location was also investigated as shown in Fig. 5 and Fig. 6. As expected, the ER was maximal at the exact location of the actual dipole and decreased for increasing polar angle separation. Similarly, the results were consistent when the dipole source was placed in an azimuthal location.

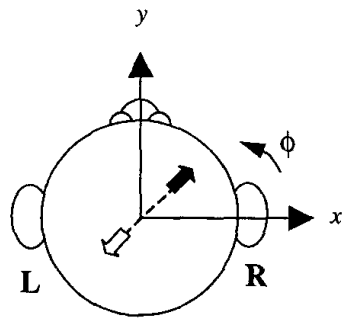

Top view

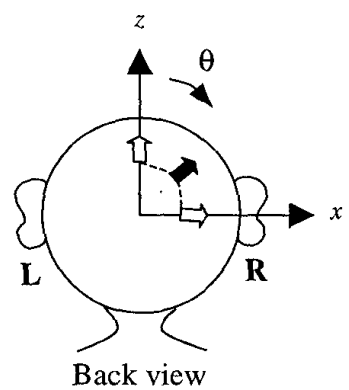

Back view
Fig. 5. Location and orientation of actual dipole (solid arrow) and estimated dipole (unfilled arrow).

\section{vi. Clinical evaluation}

A preliminary clinical evaluation of the performance of the beamformer on real EEG records was also undertaken. This comprised EEG records from 11 patients, 4 of which were graded globally by an EEGer as containing epileptiform activity and 7 as containing questionable epileptiform activity. From the first group, 12 events graded as definite or questionable EDs and from the second group, 27 events graded as questionable EDs, were selected to assess the beamformer's ability to 


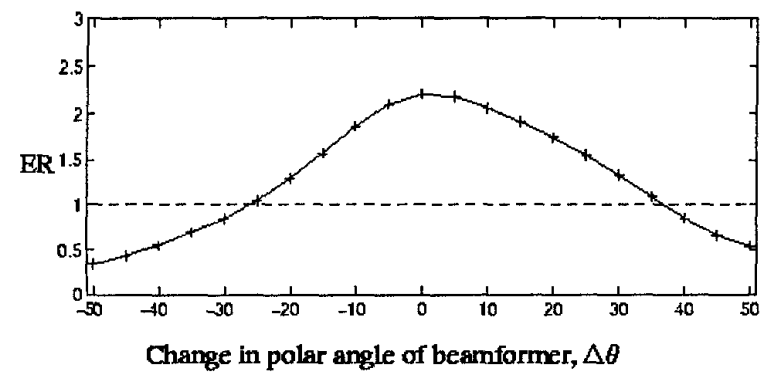

Fig. 6. Sensitivity to inaccuracies in polar location of beamformer with respect to the actual dipole.

enhance real EDs. The beamformer was found to enhance the 7 definite EDs by a mean $102 \%$ (range $54 \%$ - $215 \%$ ) with no significant difference between referential data and bipolar data. Questionable EDs (e.g., see Fig. 7) were enhanced by a mean $78 \%$ (range $3 \%-259 \%$ ).

The referential and bipolar input and beamformed projected output records of all definite and questionable EDs were randomized and shown to an EEGer for grading. As a result of beamforming, several events were upgraded from questionable to probable, indicating that they were likely to be genuine EDs, while a few were downgraded from probable to nonepileptiform indicating that they were likely to be artifacts. That notwithstanding, there was no independent means (such as depth electrodes recordings or an EEGer panel) within this preliminary study by which the validity of this upgrading - and hence the clinical effectiveness of the beamforming technique could be determined.

\section{Conclusion}

In summary, results from the simulation and preliminary clinical studies have demonstrated that the beamforming technique has considerable potential for application in clinical neurophysiology. The beamforming technique can noninvasively enhance epileptiform activity from deep foci in the brain, such as occur in mesial temporal lobe epilepsy, thereby reducing the need for alternative invasive electrophysiological investigations. The relative insensitivity of enhancement to inaccuracies in estimated location of the epileptogenic source is an important attribute of the beamformer but, conversely, indicates that other procedures are likely to be superior for localisation.

\section{REFERENCES}

[1] F. H. Lopes da Silva, A. Dijk, and H. Smits, "Detection of nonstationarities in EEGs using the autoregressive model An application to EEGs of epileptics," in CEAN: Computerized EEG analysis, H. Künkel and G. Dolce, Eds. Stuttgart: Gustav Fischer Verlag, 1975.

[2] C. J. James, M. T. Hagan, R. D. Jones, P. J. Bones, and C. J. Carroll, "Multireference adaptive noise cancelling applied to

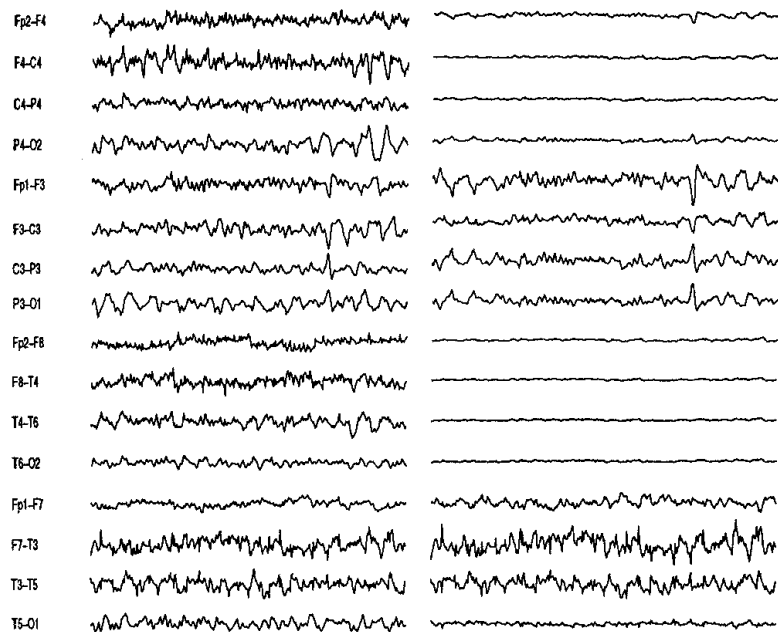

Fig. 7. A $2 \mathrm{~s}$ bipolar EEG epoch from a patient containing a questionable event showing (a) input EEG epoch and (b) projected beamformed output. The source was estimated by a volumetric search method to be at $\theta_{\mathrm{s}}=75^{\circ}, \phi_{\mathrm{s}}=170^{\circ}$, and $e_{\mathrm{s}}=0.75$ (i.e., $\sim 23 \mathrm{~mm}$ deep between C3, T3, and F3). The event was enhanced by $95 \%(\overline{\mathrm{ER}}=1.95)$.

the EEG," IEEE Trans. Biomed. Eng., vo1. 44, pp. 775-779, 1997.

[3] B. Van Veen, J. Joseph, and K. Hecox, "Localization of intra-cerebral sources of electrical activity via linearly constrained minimum variance spatial filtering," in Proc. IEEE Workshop on Stat. Sig. Array Process., 1992, pp. 526529.

[4] M. E. Spencer, R. M. Leahy, J. C. Mosher, and P. S. Lewis, "Adaptive filters for monitoring localized brain activity from surface potential time series," in Proc. 26th Asilomar Conf. Signals, Systems and Computers, 1992, pp. 156-161.

[5] W. van Drongelen, M. Yuchtman, B. Van Veen, and A. Van Huffelen, "A spatial filtering technique to detect and localize multiple sources in the brain," Brain Topogr., vol. 9, pp. 39-49, 1996.

[6] D-M. Ward, "Enhancement of deep epileptiform activity in the electroencephalogram by adaptive spatial filtering," $\mathrm{PhD}$ Thesis, University of Canterbury, Christchurch, New Zealand, 1998.

[7] L. J. Griffiths, and C. W. Jim, "An alternative approach to linearly constrained adaptive beamforming," IEEE Trans. Arr. Proc., vol. 30, pp. 27-34, 1982.

[8] Y. Salu, L. G. Cohen, D. Rose, S. Sato, C. Kufta, and M. Hallett, "An improved method for localizing electric brain dipoles," IEEE Trans. Biomed. Eng., vol. 37, pp. 699-705, 1990.

[9] S. Rush and D. A. Driscoll, "Current distributions in the brain from surface electrodes," Anaes. Analg., vol. 47, pp. 717-723, 1968. 